\title{
Enseñanza de las propiedades electrónicas y magnéticas de los compuestos de coordinación mediante fichas de actividad
}

\author{
Celia Maya díaz \\ Departamento de Química Inorgánica \\ Universidad de Sevilla \\ maya@us.es \\ ORCID: https://orcid.org/0000-0002-0651-3793 \\ D.O.I.: http://dx.doi.org/10.12795/JDU.2018.101.60 \\ Pp.: 1070-1085
}

\section{Resumen}

En esta comunicación se describe el desarrollo de una metodología de enseñanza consistente en guiar a los estudiantes en su aprendizaje a través de la resolución de una serie de fichas proporcionadas por la profesora. En estas fichas se plantean diferentes actividades consistentes en lecturas y visualización de vídeos seguidos de cuestiones teóricas y ejercicios prácticos que los alumnos deben resolver en grupos de cuatro. Con este sistema, los alumnos pueden expresar sus opiniones y razonamientos en voz alta a la vez que escuchan lo que exponen sus compañeros. En todo momento el profesor se encuentra accesible para que ellos puedan consultar todas sus dudas ayudándoles, cuando es necesario, a reconducir sus razonamientos. 
En concreto se va a aplicar dicha metodología al estudio de las propiedades electrónicas y magnéticas de los compuestos de coordinación, un tema de gran complejidad para los estudiantes de grado en Química.

Palabras Clave: Química inorgánica, Grado en Química, Docencia universitaria, Experimentación docente universitaria, Aprendizaje activo

\section{Descripción del contexto de la intervención}

El ciclo de mejora se va a aplicar en el contexto de la asignatura Química Inorgánica II del tercer curso del Grado en Química. En esta asignatura se estudia cómo son los enlaces, las estructuras, las propiedades y las reacciones químicas más importantes de los compuestos inorgánicos más representativos: los compuestos de coordinación y los sólidos inorgánicos.

- En el primer cuatrimestre se describen el enlace en los compuestos de coordinación, sus espectros electrónicos y propiedades magnéticas y los tipos de reacciones más significativas.

- En el segundo cuatrimestre se trata de conocer el enlace, la estructura, la reactividad y las propiedades de los sólidos inorgánicos.

Esta asignatura tiene carácter anual y se imparte en 2 clases semanales de 55 minutos y 8 seminarios de 2 horas cada uno que se distribuyen a lo largo del curso.

El número de alumnos matriculados es de 40 de los cuales 22 son repetidores. De ellos, 32 alumnos asisten a clase con regularidad. 


\section{Diseño previo del Ciclo de Mejora Docente (CMD)}

\section{MAPA DE CONTENIDOS}

El Ciclo de Mejora Docente se va a aplicar durante el desarrollo de 2 temas relacionados entre sí que tratan sobre las propiedades electrónicas y las propiedades magnéticas, respectivamente, de los Compuestos de Coordinación.

Los contenidos más relevantes de estos dos temas se han organizado en un mapa de de contenido que se muestra en la Figura 1:

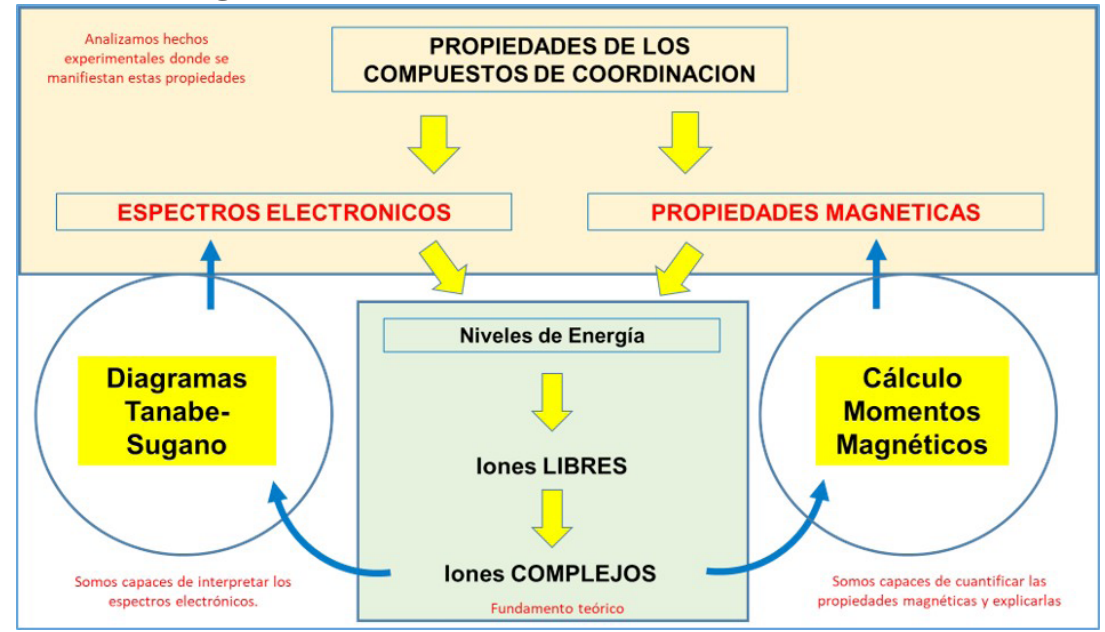

Figura 1. Mapa de contenido para las propiedades de los compuestos de coordinación.

Ambos tipos de propiedades (electrónicas y magnéticas) tienen en común un mismo fundamento teórico basado en la construcción de diagramas de niveles de energía. Aprenden en primer lugar los diagramas más sencillos correspondientes a los iones en estado libre y a continuación aprenden la construcción de esos mismos diagramas para los compuestos de coordinación que es el verdadero objetivo. A partir de esos diagramas pueden, por un lado y con ayuda de los diagramas Tanabe-Sugano, 
interpretar los espectros electrónicos de cualquier compuesto de coordinación, y por otro, calcular los momentos magnéticos efectivos de dichos compuestos y predecir o justificar sus propiedades magnéticas.

\section{MODELO METODOLOGICO}

El modelo metodológico que se plantea dentro de la estrategia docente propuesta en esta comunicación se resume en el esquema mostrado en la Figura 2:

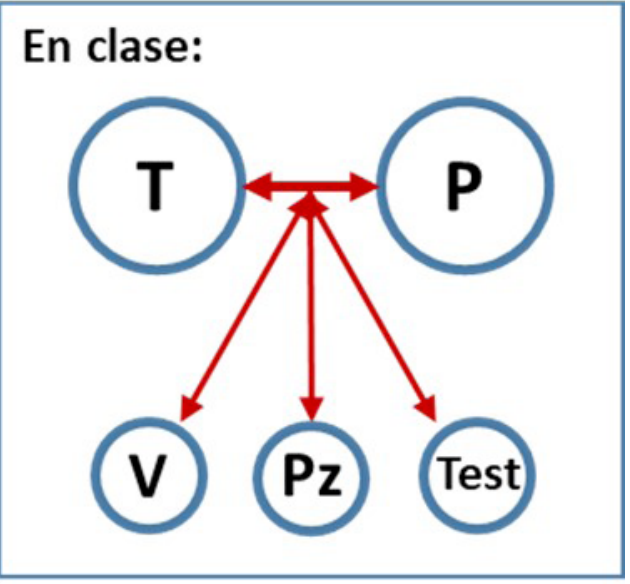

\section{En casa:}

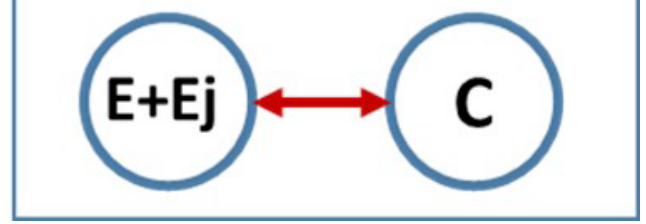

Pres $=$ Presentación $\mathbf{T}=$ Teoría $\quad \mathbf{P}=$ Práctica (Cuestiones + Ejercicios)
$\mathbf{V}=$ Vídeo
$\mathbf{P z}=$ Explicación del profesor en la pizarra
Test $=$ Test
$\mathbf{E}=$ Estudio
$\mathbf{E j}=$ Ejercicios
$\mathbf{C}=$ Corrección

Figura 2. Modelo metodológico seguido.

De acuerdo con ello, durante el desarrollo de las clases se alternan actividades en las que se presentan conceptos nuevos de teoría con actividades más prácticas. Las primeras consisten principalmente en lecturas de textos 
seleccionados o elaborados por el profesor y la visualización de vídeos de youtube. Las actividades prácticas se basan en tener que responder cuestiones relacionadas con los conceptos teóricos y su aplicación en casos prácticos. En los dos casos se pueden combinar también con explicaciones del profesor en la pizarra.

El modelo presenta también la realización de actividades en casa consistentes en estudiar los nuevos conceptos aprendidos, para afianzarlos, y la elaboración individual de ejercicios, que envían a la profesora para que se los corrija.

\section{SECUENCIA DE ACTIVIDADES PROGRAMADAS}

Para las ocho clases en las que se ha desarrollado el ciclo de mejora se programaron una serie de actividades que aparecen más abajo organizadas en tablas. Para las propiedades electrónicas se les proporciona a los alumnos 20 fichas de actividades y otras tantas para las propiedades magnéticas.

\section{Clase 1}

\begin{tabular}{|l|l|}
\hline Cuestionario & $\mathbf{1 5}^{\prime}$ \\
\hline Se pasará un cuestionario previo & \\
\hline T+P $\quad$ Tema Propiedades Electrónicas - Fichas de actividad 1-5 & $\mathbf{4 0}^{\prime}$ \\
\hline$-\quad$ Los alumnos comenzarán a trabajar en grupos de 4 con el material & \\
proporcionado por el profesor para este tema. & \\
En estas fichas se alternan la teoría con cuestiones y ejercicios, que & \\
deben ir realizando en las propias fichas. & \\
Algunas actividades se apoyan en vídeos tutoriales, que cada grupo vi- & \\
- $\begin{array}{l}\text { El prolizará en el momento que lo precise. } \\
\text { pos de alumnos. }\end{array}$ & \\
Si alguna duda es común a todos los grupos y se trata de conceptos & \\
importantes para que puedan continuar se hará una puesta en común & \\
en la pizarra. & \\
A los alumnos que no plantean dudas se les irá preguntando para te- & \\
ner la certeza de que han entendido adecuadamente los conceptos. & \\
\hline
\end{tabular}

\begin{tabular}{|l|l|c|}
\hline \multirow{2}{*}{$+\mathrm{Ej}$} & $\begin{array}{l}\text { Trabajo para casa del alumno: } \\
-\quad \text { Tendrán que realizar uno de los ejercicios de las fi- } \\
\text { chas que lo deben enviar al profesor antes de la } \\
\text { próxima clase. }\end{array}$ & $15-20^{\prime}$ \\
\hline C & $\begin{array}{l}\text { Trabajo para casa del profesor: } \\
-\quad \text { Corrección de los ejercicios. }\end{array}$ & $20-30^{\prime}$ \\
\hline
\end{tabular}


La metodología será la misma en las siguientes clases:

\section{Clase 2}

\begin{tabular}{|c|c|c|}
\hline $\mathbf{T}+\mathbf{P}$ & Tema Propiedades Electrónicas - Fichas de actividad 6-10 & $55^{\prime}$ \\
\hline \multicolumn{3}{|c|}{ Clase 3} \\
\hline $\mathbf{T}+\mathbf{P}$ & Tema Propiedades Electrónicas - Fichas de actividad 10-15 & $55^{\prime}$ \\
\hline \multicolumn{3}{|c|}{ Clase 4} \\
\hline $\mathbf{T}+\mathbf{P}$ & Tema Propiedades Electrónicas - Fichas de actividad 16-20 & $55^{\prime}$ \\
\hline \multicolumn{3}{|c|}{ Clase 5} \\
\hline $\mathbf{T}+\mathbf{P}$ & Tema Propiedades Magnéticas - Fichas de actividad 1-5 & $55^{\prime}$ \\
\hline \multicolumn{3}{|c|}{ Clase 6} \\
\hline $\mathbf{T}+\mathbf{P}$ & Tema Propiedades Magnéticas - Fichas de actividad 6-10 & $55^{\prime}$ \\
\hline \multicolumn{3}{|c|}{ Clase 7} \\
\hline $\mathbf{T}+\mathbf{P}$ & Tema Propiedades Magnéticas - Fichas de actividad 11-15 & $55^{\prime}$ \\
\hline \multicolumn{3}{|c|}{ Clase 8} \\
\hline $\mathbf{T}+\mathbf{P}$ & Tema Propiedades Magnéticas - Fichas de actividad 16-20 & $55^{\prime}$ \\
\hline
\end{tabular}

Finalmente, los alumnos enviarán al profesor todas las actividades realizadas a lo largo de las clases. A continuación, el profesor publicará los resultados de esas actividades y entonces los alumnos deben devolver el tema al profesor comentando aquellos aspectos que tras la lectura de las respuestas del profesor les hayan hecho reflexionar, darse cuenta de lo que no han entendido, etc.

\section{CUESTIONARIO PREVIO}

El cuestionario que se prepara para pasárselo a los alumnos el primer día del ciclo de mejora se presenta a continuación:

1.- Considera un complejo de un metal de configuración d ${ }^{1}$ e indica cuántos picos observarás en su espectro UV-Visible. Justifica tu respuesta dibujando sobre un esquema de energía las transiciones que corresponden a esos picos.

Jornadas de Formación e Innovación Docente del Profesorado I № 1 (2018) Esta obra se distribuye con la licencia Creative Commons Reconocimiento-NoComercial-SinObraDerivada Internacional (CC BY-NC-ND 4.0.) 
2.- Considera un complejo de un metal de configuración d $d^{2}$ e indica cuántos picos observarás en su espectro UV-Visible. Justifica tu respuesta dibujando sobre un esquema de energía las transiciones que corresponden a esos picos.

3.- ¿Qué crees que necesitas conocer para interpretar un espectro UV-visible?

4.- Enumera los distintos tipos de sustancias que conoces de acuerdo a sus propiedades magnéticas e indica cuáles son estas propiedades.

5.- Explica por qué se asocia el paramagnetismo a la existencia de electrones desapareados.

\section{Aplicación del CMD}

\section{Clase 1.}

Al comienzo de la clase se pasa a los alumnos el cuestionario previo y, a continuación, comienzan a trabajar con las primeras fichas en las que se les presentan una serie de casos prácticos (ver ejemplo en la Figura 3). Dichos casos consisten todos ellos en proporcionarles ejemplos de compuestos cuyos espectros electrónicos deben predecir, o bien, se les da el espectro y lo deben justificar. La dificultad de los casos va en aumento.

Ejemplo 1: complejo $\left.\left[\mathrm{Ti}_{(\mathrm{H}} \mathrm{H}_{2} \mathrm{O}\right)_{6}\right]^{3+}$ Configuración dn del ion metálico:

Este es su espectro UV-Visible:

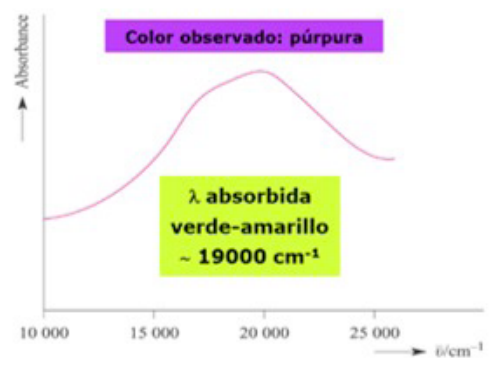

¿Cómo puedes explicar el espectro de ese complejo? ¿Por qué tiene una única banda?

Figura 3. Ejemplo de caso práctico propuesto al comienzo del tema.

Jornadas de Formación e Innovación Docente del Profesorado | № 1 (2018)

Esta obra se distribuye con la licencia Creative Commons 
En este momento, para resolver los casos planteados los alumnos emplean los únicos diagramas de niveles de Energía que conocen y comienzan a hacer sus predicciones. Al ir avanzando en los casos van comprobando que sus pronósticos no coindicen con los espectros experimentales que se les van mostrando y comienzan a caer en la cuenta de que algo de lo que están haciendo es incorrecto. El error está en los diagramas de energía que utilizan que no son los adecuados para interpretar espectros electrónicos.

En sus discusiones y argumentaciones, al trabajar en los grupos, se detecta que tienen errores de conceptos básicos importantes, ya que, incluso al utilizar los diagramas de niveles de energía que conocen, hacen razonamientos erróneos acerca de lo que éstos significan.

La clase se cierra con la puesta en común de los resultados y la toma de conciencia de que los diagramas de energía que han venido utilizando hasta ahora no sirven a la hora de interpretar espectros electrónicos. Esos diagramas sólo representan una situación posible de las moléculas, pero en realidad existen muchas situaciones de idéntica energía para una misma molécula y en una muestra se van a dar todas esas posibles situaciones que deben considerar.

\section{Clase 2}

Los alumnos siguen trabajando con las siguientes fichas donde se les empieza a enseñar cómo construir los nuevos diagramas de energía. En primer lugar, van construyendo los diagramas de los iones metálicos libres y para ello necesitan conocer una serie de conceptos nuevos que se les van presentando y cuyo significado deben ir buscando en la bibliografía. Resulta que recientemente han estudiado muchos de esos conceptos en otra asignatura (Química Física) y ahora le ven por primera vez una 
aplicación. En esta clase, por tanto, avanzan más rápidamente de lo esperado.

La clase concluye y la mayoría de los alumnos sabe cómo construir los diagramas para los iones metálicos libres.

\section{Clase 3.}

Las nuevas fichas plantean la construcción de los diagramas de niveles de energía para los compuestos de coordinación (iones + ligandos unidos a ellos).

A los alumnos les cuesta mucho comprender lo que hacen ahora. Trabajan en los casos propuestos y, aunque parece que avanzan, la impresión es que no están entendiendo lo que están haciendo ni para qué lo hacen exactamente. De manera que en este punto han perdido el hilo del tema.

La clase se concentra entonces en que aprendan a hacer esos diagramas aunque no entiendan aún para qué los necesitan.

Esta vez no terminan todas las tareas propuestas para la clase y se dejan para el día siguiente.

\section{Clase 4.}

En esta clase siguen trabajando en la construcción de los diagramas de los compuestos de coordinación. Cuando la mayoría de los grupos ha terminado las tareas se dedica la última parte de la clase a que la profesora presente un esquema en el que les ha resumido los contenidos más importantes de la parte del tema referida a los espectros electrónicos (Figura 4). Entonces se les explica cuál es el objetivo de aprender a construir los nuevos diagramas de energía. Para que realmente puedan entenderlo se realiza con ellos un ejemplo práctico en la pizarra. 


\section{Clase 5.}

En esta clase los alumnos comienzan a trabajar en la parte del tema dedicada a las propiedades magnéticas de los compuestos de coordinación.

En primer lugar, se visualizan dos vídeos de 5 minutos cada uno. El primero de ellos trata de propiedades magnéticas en general: qué son, a qué se debe que un compuesto las tenga o no, qué tipos de sustancias existen según sus propiedades magnéticas, cómo se comportan en presencia de un campo magnético... en definitiva conceptos muy generales.

En el segundo vídeo se entra más en profundidad en los tipos de propiedades magnéticas que les interesan. A partir de esos vídeos los alumnos conocen los tipos de sustancias que existen y describen sus propiedades magnéticas.

Objetivo Principal SABER INTERPRETAR ESPECTROS

ELECTRONICOS DE LOS

COMPLEJOS DE LOS METALES DE

TRANSICIÓN

¿Qué necesito saber?

Cuantas transiciones de distinta energía se pueden dar en un

determinado complejo.

En su espectro se van a observar tantos picos como transiciones se puedan dar.

¿Cómo puedo conocer

las transiciones que se pueden dar en un complejo?

Dibujando su esquema de niveles de energía.

Las transiciones se darán entre el nivel fundamental y los niveles excitados.

(Deben cumplirse las reglas de selección).

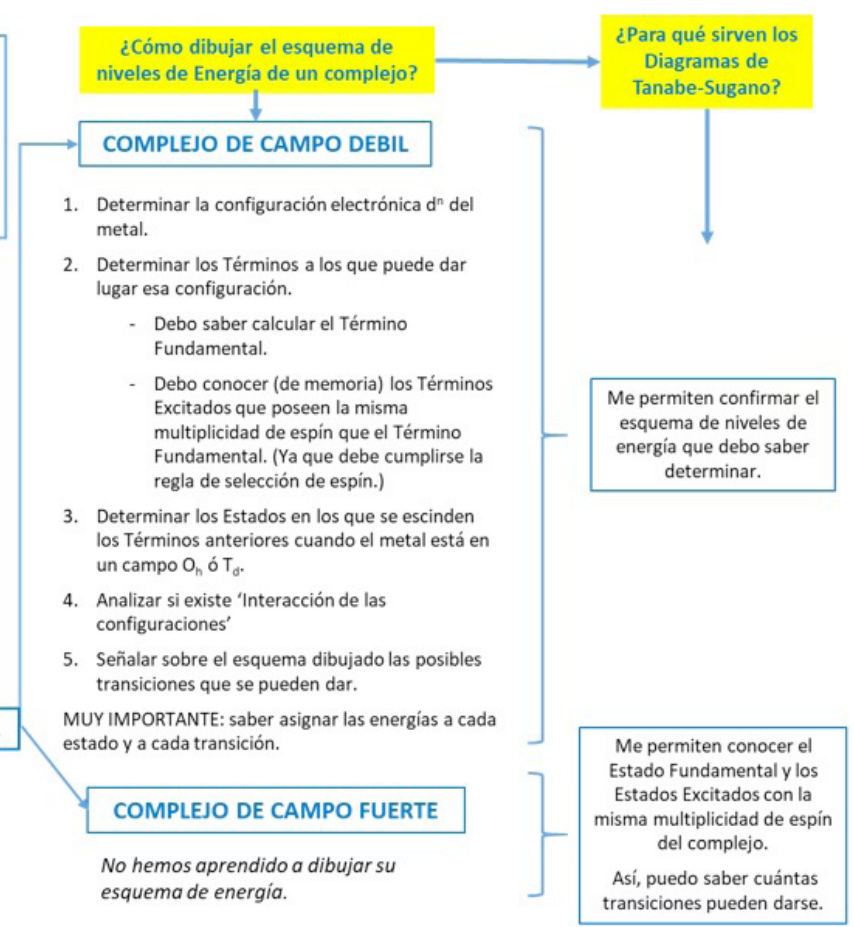

Figura 4. Resumen de los contenidos del tema relacionados con las propiedades electrónicas de los compuestos de coordinación. 
A continuación, investigan cómo se puede medir el magnetismo experimentalmente. Todos ellos describieron el método de la Balanza de Gouy sin demasiadas dificultades y entendieron el significado de la propiedad 'Susceptibilidad Magnética'.

\section{Clase 6.}

Se continúa trabajando con el concepto de Susceptibilidad Magnética y los alumnos acaban deduciendo que la susceptibilidad es un parámetro que varía con la temperatura y que resulta más práctico definir una propiedad que no dependa de la temperatura, que se va a llamar momento magnético efectivo.

En este punto los alumnos entienden que el siguiente objetivo, que es el objetivo central del tema, es aprender a calcular el momento magnético efectivo en cualquier compuesto de coordinación y que a través de él se podrán establecer su comportamiento magnético.

Aprenden cómo se calcula este parámetro en los iones metálicos libres.

\section{Clase 7.}

En esta clase los estudiantes comienzan a aprender las diferentes formas de calcular el momento magnético en los compuestos de coordinación y como están directamente relacionadas con los diagramas de niveles de energía que aprendieron en las primeras clases del ciclo.

Establecer esa relación es muy complejo y los alumnos aprenden a hacer el cálculo del momento magnético efectivo, pero no consiguen entender los diagramas de energía que justifican ese cálculo en cada caso. 


\section{Clase 8.}

Esta última clase se desarrolla de una manera distinta a la programada. La profesora presenta un resumen en una diapositiva y en la pizarra va construyendo uno a uno cada tipo de diagrama de niveles de energía contando en todo momento con la aportación de los alumnos.

\section{TABLA RESUMEN DE CÓMO CALCULAR $\mu_{e t}$}

$\mu_{J, L, S}$

- Iones lantánidos libres

$$
\mu_{J, L, S}=g \sqrt{J(J+1)}
$$

$\mu_{S}$

- Complejos de $M_{T}$ con TF A ó E

- Complejos de $M_{T}$ de la $1^{\text {a }}$ serie con TF T

(acoplamiento L-S poco importante)

$$
\mu_{e f}=\sqrt{n(n+2)} \quad \mu_{S}=2 \sqrt{S(S+1)}
$$

n: número de e-desapareados

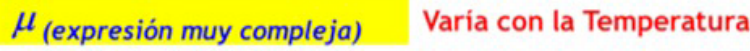

- Complejos de $M_{T}$ de la $2^{\mathrm{a}}$ y $3^{\mathrm{a}}$ serie con TF T

(acoplamiento L-S muy importante)

- Iones libres de $M_{T}$

Figura 5. Resumen de los contenidos del tema relacionados con las propiedades magnéticas de los compuestos de coordinación.

\section{Clase 9.}

En la clase siguiente al ciclo de mejora se pasa de nuevo el mismo cuestionario inicial durante los primeros 15 minutos.

\section{Evaluación del CMD}

\section{EVALUACIÓN DEL APRENDIZAJE DE LOS ESTUDIANTES}

A partir del cuestionario previo que se pasó a los alumnos al comienzo del ciclo de mejora se pudo establecer el punto de partida de todos los alumnos de la clase. Para cada pregunta se establecieron las correspondientes escaleras de aprendizaje y evaluación. Tras la realización de 
todas las actividades programadas para el ciclo de mejora los alumnos volvieron a realizar el mismo cuestionario construyéndose nuevas escaleras. (Figura 6).

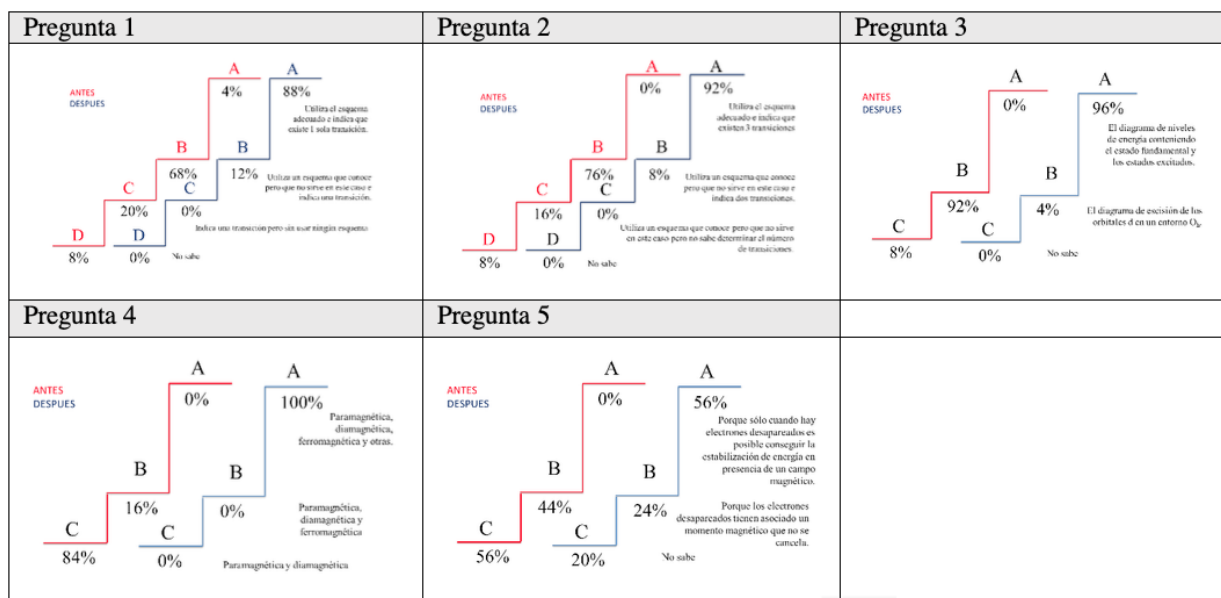

Figura 6. Escaleras d££e aprendizaje para cada una de las preguntas del cuestionario.

La correlación de los cuestionarios iniciales y finales fue analizada en 25 sujetos elegidos al azar, obteniéndose los resultados que se muestran en la Tabla 1.

\begin{tabular}{|c|c|c|c|c|c|c|c|c|c|c|c|c|c|c|c|}
\hline Evaluación & \multicolumn{15}{|c|}{ Preguntas $\left(1^{\mathrm{a}}\right.$ columna: ANTES, $2^{\mathrm{a}}$ columna: DESPUES $)$} \\
\hline Sujeto & 1 & 1 & & 2 & 2 & & 3 & 3 & & 4 & 4 & & 5 & 5 & \\
\hline 1 & D & B & $\uparrow \uparrow$ & D & B & $\uparrow \uparrow$ & $\mathrm{C}$ & A & $\uparrow \uparrow$ & C & A & $\uparrow \uparrow$ & B & B & $\leftrightarrow$ \\
\hline 2 & D & A & $\uparrow \uparrow \uparrow$ & $\mathrm{C}$ & B & $\uparrow$ & B & B & $\leftrightarrow$ & $\mathrm{C}$ & A & $\uparrow \uparrow$ & $\mathrm{C}$ & $\mathrm{C}$ & $\leftrightarrow$ \\
\hline 3 & C & B & $\uparrow$ & D & A & $\uparrow \uparrow \uparrow$ & B & A & $\uparrow$ & C & A & $\uparrow \uparrow$ & B & A & $\uparrow$ \\
\hline 4 & $\mathrm{C}$ & A & $\uparrow \uparrow$ & B & A & $\uparrow$ & B & $\mathrm{A}$ & $\uparrow$ & C & A & $\uparrow \uparrow$ & C & B & $\uparrow$ \\
\hline 5 & C & A & $\uparrow \uparrow$ & C & A & $\uparrow \uparrow$ & B & A & $\uparrow$ & C & A & $\uparrow \uparrow$ & B & A & $\uparrow$ \\
\hline 6 & C & A & $\uparrow \uparrow$ & B & A & $\uparrow$ & B & A & $\uparrow$ & C & A & $\uparrow \uparrow$ & C & A & $\uparrow \uparrow$ \\
\hline 7 & C & A & $\uparrow \uparrow$ & B & A & $\uparrow$ & B & A & $\uparrow$ & B & A & $\uparrow$ & C & C & $\leftrightarrow$ \\
\hline 8 & B & A & $\uparrow$ & B & A & $\uparrow$ & B & A & $\uparrow$ & C & A & $\uparrow \uparrow$ & C & C & $\leftrightarrow$ \\
\hline 9 & B & A & $\uparrow$ & B & A & $\uparrow$ & B & A & $\uparrow$ & C & A & $\uparrow \uparrow$ & C & B & $\uparrow$ \\
\hline 10 & B & $\mathrm{A}$ & $\uparrow$ & B & A & $\uparrow$ & B & A & $\uparrow$ & C & A & $\uparrow \uparrow$ & C & A & $\uparrow \uparrow$ \\
\hline 11 & B & A & $\uparrow$ & B & A & $\uparrow$ & B & A & $\uparrow$ & C & A & $\uparrow \uparrow$ & C & B & $\uparrow$ \\
\hline 12 & B & A & $\uparrow$ & B & A & $\uparrow$ & B & $\mathrm{A}$ & $\uparrow$ & C & A & $\uparrow \uparrow$ & B & B & $\leftrightarrow$ \\
\hline 13 & B & B & $\leftrightarrow$ & B & A & $\uparrow$ & B & A & $\uparrow$ & C & A & $\uparrow \uparrow$ & B & A & $\uparrow$ \\
\hline 14 & B & A & $\uparrow$ & C & A & $\uparrow \uparrow$ & C & A & $\uparrow \uparrow$ & C & A & $\uparrow \uparrow$ & B & A & $\uparrow$ \\
\hline 15 & B & A & $\uparrow$ & B & A & $\uparrow$ & B & A & $\uparrow$ & B & A & $\uparrow$ & C & A & $\uparrow \uparrow$ \\
\hline 16 & B & A & $\uparrow$ & B & A & $\uparrow$ & B & A & $\uparrow$ & C & A & $\uparrow \uparrow$ & B & A & $\uparrow$ \\
\hline 17 & B & A & $\uparrow$ & B & A & $\uparrow$ & B & A & $\uparrow$ & C & A & $\uparrow \uparrow$ & C & A & $\uparrow$ \\
\hline 18 & B & A & $\uparrow$ & B & A & $\uparrow$ & B & A & $\uparrow$ & B & A & $\uparrow$ & C & C & $\leftrightarrow$ \\
\hline 19 & B & A & $\uparrow$ & B & A & $\uparrow$ & B & A & $\uparrow$ & C & A & $\uparrow \uparrow$ & C & A & $\uparrow \uparrow$ \\
\hline 20 & B & A & $\uparrow$ & B & A & $\uparrow$ & B & A & $\uparrow$ & C & A & $\uparrow \uparrow$ & C & A & $\uparrow \uparrow$ \\
\hline 21 & B & A & $\uparrow$ & B & A & $\uparrow$ & B & A & $\uparrow$ & C & A & $\uparrow \uparrow$ & B & A & $\uparrow$ \\
\hline 22 & B & A & $\uparrow$ & C & A & $\uparrow \uparrow$ & B & A & $\uparrow$ & B & A & $\uparrow$ & C & C & $\leftrightarrow$ \\
\hline 23 & B & A & $\uparrow$ & B & A & $\uparrow$ & B & A & $\uparrow$ & C & A & $\uparrow \uparrow$ & B & B & $\leftrightarrow$ \\
\hline 24 & B & A & $\uparrow$ & B & A & $\uparrow$ & B & A & $\uparrow$ & C & A & $\uparrow \uparrow$ & B & A & $\uparrow$ \\
\hline 25 & A & A & $\leftrightarrow$ & B & A & $\uparrow$ & B & A & $\uparrow$ & C & A & $\uparrow \uparrow$ & B & A & $\uparrow$ \\
\hline
\end{tabular}

Tabla 1. Análisis de los cuestionarios iniciales y finales de 25 alumnos elegidos al azar. Jornadas de Formación e Innovación Docente del Profesorado I № 1 (2018) Reconocimiento-NoComercial-SinObraDerivada Internacional (CC BY-NC-ND 4.0.) 
El análisis conjunto de las escaleras y de los resultados mostrados en la tabla revela que prácticamente todos los alumnos alcanzaron el nivel de aprendizaje deseado en relación con los espectros electrónicos de los compuestos de coordinación. En cuanto a la parte correspondiente a las propiedades magnéticas el nivel de aprendizaje fue algo menor. Si bien todos los alumnos aprendieron los tipos de propiedades magnéticas que se pueden encontrar en estos compuestos, sólo un 56\% consiguió entender perfectamente por qué la propiedad del paramagnetismo se da únicamente si hay electrones desapareados en el compuesto, una relación que casi todos conocen de cursos anterior pero que dan por sentado sin llegarse a cuestionar por qué es realmente así. Es cierto que la explicación es muy compleja y larga. Tal vez por su longitud acaban perdiendo la conexión entre el primer concepto y el último.

\section{EVALUACIÓN DEL DISEÑO DEL CICLO DE MEJORA}

Una vez finalizado el ciclo de mejora y realizada la evaluación de los alumnos se pueden valorar los aspectos positivos y negativos del mismo. Varios son los aspectos positivos destacables. Desde un punto de vista docente ha resultado muy efectivo reflexionar previamente acerca de los contenidos del tema y tratar de concentrarlos todos en un único esquema (mapa de contenido). A partir de ahí se ha podido realizar con más facilidad el diseño del tema y la organización de las diferentes actividades docentes. Tener un plan perfectamente organizado de las actividades a desarrollar en las clases aporta una mayor seguridad al profesor ya que todos los tiempos están establecidos y no se deja nada a la improvisación. Además, la realización de los cuestionarios al inicio y al comienzo del ciclo de mejora ha permitido conocer el punto de partida y el punto final de la globalidad de los alumnos, habiendo podido cuantificar el grado de éxito del modelo docente aplicado. Otro aspecto muy positivo de este ciclo mejora ha sido el 
trabajo de los alumnos en grupo. Han realizado tareas de indole teórica o práctica, de menor a mayor dificultad, enfrentándose a conceptos nuevos para ellos, y con el apoyo de sus compañeros y de la profesora han podido resolver los obstáculos y alcanzar los objetivos, de manera guiada pero bastante autónoma. El grado de implicación y de satisfacción de los alumnos es realmente notable.

Por otro lado, a medida que el ciclo de mejora se ha ido desarrollando han podido observarse aspectos mejorables: actividades que deben modificarse porque los alumnos no han entendido su utilidad dentro del discurrir del tema o que no estaban bien planteadas, actividades que deben implementarse para mejorar la comprensión de ciertos conceptos o el ajuste del tiempo empleado en las diversas actividades.

\section{Conclusiones}

En esta comunicación se ha presentado el diseño y la aplicación de un ciclo de mejora al tema Propiedades electrónicas y magnéticas de la asignatura Química Inorgánica Il del tercer curso del Grado de Química. El diseño propuesto supone una nueva manera de enseñar una serie de conceptos muy complejos, de difícil comprensión para los alumnos y que tradicionalmente son explicados en clases magistrales sin que los alumnos tengan la oportunidad de reflexionar con el tiempo necesario y en presencia del profesor acerca de esos nuevos conceptos.

La metodología aplicada, consistente en la realización de fichas de actividades por los alumnos, en grupo y con la asistencia del profesor, se ha demostrado muy satisfactoria, como ha podido comprobarse mediante el proceso de evaluación llevado a cabo a través de cuestionarios.

Los extraordinarios resultados obtenidos, con prácticamente la totalidad de los alumnos alcanzando los objetivos propuestos, representan el mejor argumento para reconocer el interés de este tipo de metodología docente 
en la que los alumnos pasan de ser sujetos pasivos que reciben información del profesor a ser los responsables directos de su propio aprendizaje.

\section{Bibliografía}

Bain, K. (2007). Lo que hacen los mejores profesores de universidad. Valencia: Universitat de Valencia.

Finkel, D. (2008). Dar clase con la boca cerrada. Valencia: Universitat de Valencia.

Porlan, R. (2017). Enseñanza universitaria. Cómo mejorarla. Ediciones Morata, S. L.

C. E. Housecroft \& A. G. Sharpe (2012) Inorganic Chemistry. $4^{\text {th }}$ Ed., Pearson, Prentice Hall

P. W. Atkins, T. Overton, J. Rourke, M. Weller \& F. Armstrong (2013) Inorganic Chemistry. $5^{\text {th }}$ Ed. McGraw-Hill.

Jornadas de Formación e Innovación Docente del Profesorado | № 1 (2018) Esta obra se distribuye con la licencia Creative Commons 